\title{
Multiple Optimal Path Identification USING ANT COLONY OPTIMISATION IN WIRELESS SENSOR NETWORK
}

\author{
Aniket. A. Gurav ${ }^{1}$, Manisha. J. Nene ${ }^{2}$ \\ Department of Applied Mathematics ${ }^{1}$ \\ Department of Applied Mathematics and Computer Engineering ${ }^{2}$ \\ Defence Institute of Advance Technology ${ }^{12}$, Pune, India 411025
}

\begin{abstract}
Wireless Sensor Network WSN is tightly constrained for resources like energy, computational power and memory. Many applications of WSN require to communicate sensitive information at sensor nodes SN to Base station BS. The basic performance of WSN depends upon the path length and numbers of nodes in the path by which data is forwarded to BS. In this paper we present bio-inspired Ant Colony Optimisation ACO algorithm for Optimal Path Identification OPI for packet transmission to communicate between SN to BS. Our modified algorithm OPI using ACO is base-station driven which considers the path length and the number of hops in path for data packet transmission. This modified algorithm finds optimal path OP as well as several suboptimal paths between $S N \& B S$ which are useful for effective communication.
\end{abstract}

\section{KEYWORDS}

WSN, Ad-Hoc Network, ACO, Optimal path Identification, Multiple optimal paths

\section{INTRODUCTION}

A WSN is an ad-Hoc network composed of hundreds or even thousands of SN. These SN are capable of sensing. SN's are battery powered. Every time replacing or recharging of battery is not possible. The SN's have to perform tasks such as object monitoring \& tracking, detecting the presence of certain objects, event monitoring, data fusion \& localization. These tasks of SN generate a vast amount of data. The SN has to forward this data to BS (sink node).It is found that SN consumes lot of energy while communicating this information so over all network lifetime will increase if WSN consumes minimum energy in communication. The reporting between SN \& BS consumes energy based on the type of communication protocol, communication path \& number of hops between SN and BS. This requires finding out optimal path OP between BS to SN which will be useful in efficiently forwarding data, reducing delay, evenly distributing traffic load \& power consumption and communication overhead in WSN.

In our work we are focused on identifying optimal and suboptimal communication path between $\mathrm{SN}$ and BS.

There are various routing protocols like Direct diffusion [9], LEACH (Low Energy Adaptive Clustering Hierarchy) [8], SPIN (Sensor protocols for Information via Negotiation) [8] [10].The Shortest path finding is the backbone for the routing algorithms in WSN. So it is a graph based problem to find out the shortest path between two vertices. As WSN is resource constrained it is not desirable to use the conventional shortest path finding techniques.

DOI : 10.5121/ijwmn.2013.5508 
So we should look at soft computing techniques. It can help us in dynamically changing environment for setting communication paths in WSN. ACO is a soft computing technique for Optimisation. This method is having characteristic like achieving global optimization through local interaction, high degree of self organization and adaptiveness.

\subsection{Motivation}

Routing in WSN is challenging due to limited capabilities of $\mathrm{SN}$, dynamically changing communication network environment and network topology, abrupt failure of SN etc. To handle these many routing techniques exist, such as data centric, location based and hierarchical routing. Finding shortest path in WSN during routing can help to optimise communication and computation overhead.

In target or object tracking, surveillance vast amount of data is generated. This data should be forwarded to BS with an optimal path to process it in the real time. A Greedy algorithm like Dijkstra's algorithm, Bellman Ford and Dynamic programming algorithm are useful in shortest path finding but it is having high computational complexity. Greedy algorithm doesn't give a guarantee of finding the globally OP and these techniques is helping to identify a single static shortest path but the only single shortest path is not desirable for WSN.

Bio-inspired shortest path algorithm using ACO proves to be efficient in the OP detection. By studying traditional ACO we found its following drawback for WSN which are possible to overcome. Firstly ACO based routing [1] [4] algorithm which is the present finds path by creating ants in the form of data packets, Data packets are transmitted between each node i.e. peer to peer. This increases the communication overhead which is likely to result in increased network traffic. Also packet collision and packet loss can result decrease in efficiency.

Existing routing protocols can be improved by considering the path length and the number of nodes in path as a critical parameter to detect the optimal path between SN to BS. Secondly the traditional ACO [11] considers only path length not the number of hops in the path and it is not guaranteed to lead the optimal solution every time. Considering these challenges we have modified the Traditional ACO in an appropriate way .Our work overcomes above mentioned issues \& BS computes the OP and communicates it with the SN. So in our approach path discovery is done prior to the data transmission.

\subsection{OVERVIEW OF OUR WORK}

In our work we are proposing BS driven OPI. Here BS is made aware (directly or indirectly) about the all SN their position and topology of the network. So problem of finding the optimal path now becomes graph based problem. 


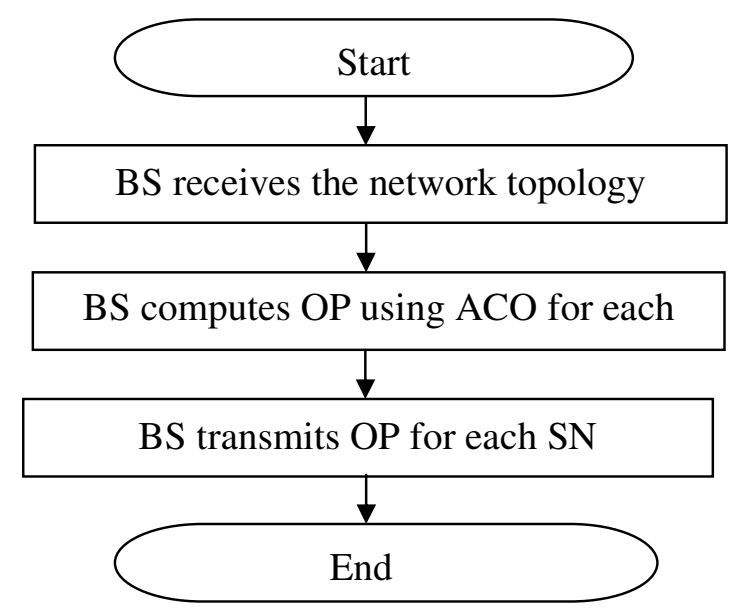

FIG. 1 SHOWS OVERVIEW OF OUR WORK

Here BS calculates OP using ACO \& communicates that path with respective SN. So in $1^{\text {st }}$ step BS made aware about the network topology. In $2^{\text {nd }} \mathrm{OP}$ is identified. Then in the 3rd step OP is communicated with the respective SN. After all these steps are done then any SN who wants to send data to BS can use above calculated OP.

\subsection{ASSUMPTIONS \& EXPERIMENT PARAMETERS}

For applying ACO for finding OP our assumptions are as follows.

\section{a) Assumption at BS \& SN}

BS is aware about co-ordinate of all SN present and so it is also aware about the topology of the entire WSN. BS is responsible for the entire computation of ACO. These are assumed about BS. In case of SN it is assumed that SN's are already localized. All SN's are static. SN's cannot be recharged. Communication range of all $\mathrm{SN}$ is same.

\section{b) Assumption for OPI Using ACO}

Traditional ACO is explained in this paper, for our experiment we have taken the value of parameters $\alpha=1, \beta=3, \rho=0.2$. In our experiment WSN is treated as a graph. All the SN's which are in communication range of each other are represented as adjacent nodes in the graph. $\theta$ is no of nodes (hops) in the path from source to destination. $\Omega$ a is a parameter which manages relative importance of path length \& $\psi$ controls the importance of nodes in path (equation 6). The value of these two variables is 3 .

\section{RouTing USING ACO}

Routing using ACO is a complex task due to resource constrained WSN. In routing SN node route data to BS using the most efficient path. In ACO based approach behaviour of real ant searching for food through pheromone deposition helps to find the optimal path. 


\subsection{Routing using Traditional ACO}

When ants trace out a path from their nest to a food source, ant drop chemical called pheromone on the path. On the shorter path more pheromone gets accumulated. This is because shorter paths accumulate pheromone deposits at a faster rate. Suppose each ant starts from the source "s" to destination " $d$ ", it tries to find the shortest path between these nodes. At each node " $i$ " ant " $k$ " decides to visit the next node " $j$ " based upon the probability given by formulae in equation 1 .

$$
p_{i j}^{k}=\left(\begin{array}{cc}
\frac{\left[\tau_{i j}\right]^{\alpha} *\left[\eta_{i j}\right]^{\beta}}{\sum\left[\tau_{i j}\right]^{\alpha} *\left[\eta_{i j}\right]^{\beta}} & \forall j \in N_{j} \& j \notin M^{k} \\
0 & \text { otherwise }
\end{array}\right)
$$

Where $\tau_{i j}$ is the pheromone concentration on edge between node " $i$ " to node " $j$ ". $\eta_{i j}$ is the value of heuristic related to path length, $\alpha$ and $\beta$ are two parameters which controls the relative importance of pheromone trail and heuristic value related to path length. $N_{j}$ is set of SN. $M^{k}$ Is the memory of ant " $\mathrm{k}$ " The heuristic value related to path length is

$$
\eta_{i j}=\frac{1}{l(i, j)}
$$

Where $l(i, j)$ is the edge length between nodes "i" and " $j$ ".

After each iteration " $t$ ", ants " $k$ " deposit quantity of pheromone which is given by

$$
\Delta \tau^{k}(t)=\left(1 / j^{k}(t)\right)
$$

Where $j^{k}(t)$ is the length of the path from source to destination traversed by ant "k". Total Amount of pheromone quantity on edge " $i$ " to " $j "$ is given by the equation

$$
\tau_{i j}(t) \leftarrow \tau_{i j}(t)+\Delta \tau_{i j}(t)
$$

With increase in number of iteration/time parameter the pheromone starts evaporating. A control coefficient $\rho \in[0,1]$ decides the amount of pheromone on each edge at any specific iteration which is given by the equation

$$
\tau_{i j}(t) \leftarrow(1-\rho) \tau_{i j}(t)
$$

As the number of iteration increases pheromone on shortest path becomes more as compared to other paths present in the WSN. So the number of ants gets attracted to shorter path. Finally pheromone concentration on this path becomes higher and algorithm converges.

\subsection{OPI USING ACO}

Steps 1 to 7 describes the working of our proposed algorithm. Our proposed algorithm is modified according to the needs of energy constrained WSNs. The proposed algorithm considers above mentioned two parameters path length and number of hops in path to find out OP.

\section{Steps}

Step 1: When the BS starts computing shortest path, it decides one destination node in 
International Journal of Wireless \& Mobile Networks (IJWMN) Vol. 5, No. 5, October 2013

WSN. An ant is created which will generate a path from BS to that destination node.

Step 2: Ant " $k$ " at node " $i$ " selects the next node " $j$ " using formula in equation 1.

Here " $j$ " is one of the adjacent node of " $i$ ". An ant " $k$ " has more probability to choose the node with larger values of $p_{i j}^{k}$ the next node selected is stored in memory of ant k denoted by $\left(M^{k}\right)$.

Step 3: If any ant visits the node which is already visited by the same ant then that ant Is discarded from path calculation.

Step 4: Step 2 and 3 are repeated until ant "k" finds its destination node or discarded.

Step 5: Step 1 to 4 is repeated for all ants.

Step 6: When all ants complete above procedure pheromone on the edge is updated which is given by equation 6 .

$$
\tau_{i j}(t) \leftarrow \tau_{i j}(t)+\left(\left(\frac{1}{\theta^{\Psi}}\right) \times\left(1+\left(\frac{1}{j^{k}(t)^{\Omega}}\right)\right)\right.
$$

Step 7: Then the evaporation of pheromone is calculated by equation 5 .

Step 8: Now the path traversed by all ants is compared. The best optimal path is selected Among them by comparing the number of hops \& distances. This optimal The path is then deleted from the graph. Again same procedure is repeated, this time result will be a different optimal path than the previous one because that path is already deleted.

As the above steps are iterated, BS comes to know about the optimal \& suboptimal paths between it and the destination. Similarly the BS can calculate distance between it and all other SN.

\subsection{RESULT}

This section compares the result of optimal path found using modified ACO and traditional ACO. Figures 2,3 and 4 shows deployed WSN and its communication network. The nodes which can directly communicate is shown by edges. The distance between corresponding $\mathrm{SN}$ is indicated near the edges. 

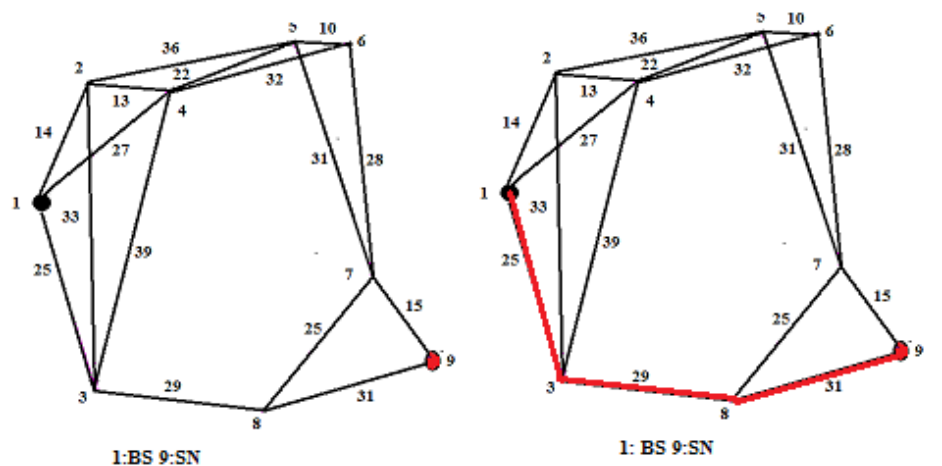

Fig. 2 Wireless Sensor Nodes and its network Fig. 3 OP using modified ACO

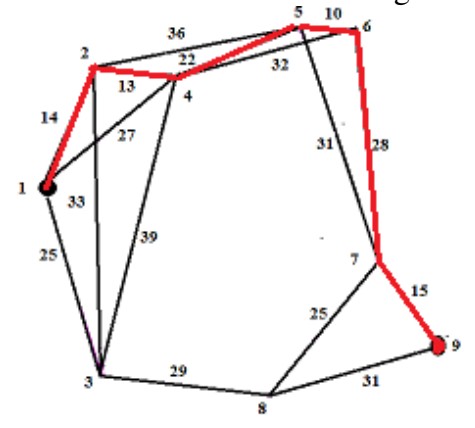

1: BS 9: SN

Fig. 4 Optimal Path using traditional ACO

In the above scenario of figure 1 node 9 senses some event and it want to forward that information to node 1 . Node 1 which is BS is aware about co-ordinates and the topology of the entire network .It implements proposed algorithm and finds the optimal paths between SN 1 and 9. This step is repeated for all nodes and optimal path found is communicated with all SN. So all SN including 9 is now aware about the optimal path between itself to BS. In this way finding the optimal paths is done. So every SN know how to forward data to BS. The initial pheromone concentration on all edges is as shown in table number 1.The left most columns and top row denote the node numbers. Here $\mathrm{N}$ in top most left block in table 1 stands for node each edge is initialised with pheromone quantity 0.00001 . This is initialisation necessary to begin the execution of ACO. This pheromone quantity is kept as small as possible because it should not affect the final result. As the ants traverse the path, pheromone concentration on that edge start increasing. Finally more pheromone gets deposited on the path with the shorter length, which is overused. This converges to the optimal path. After implementing the modified algorithm the pheromone concentration in a 5th iteration is shown in table 2. Here left column and top row denote the node number and $\mathrm{N}$ in top most left block in table 2 represents the sensor node. 
Table 1 Pheromone value

\begin{tabular}{|l|l|l|l|l|l|l|l|l|l|}
\hline $\mathrm{N}$ & 1 & 2 & 3 & 4 & 5 & 6 & 7 & 8 & 9 \\
\hline 1 & 0 & 0.00001 & 0.00001 & 0.00001 & 0 & 0 & 0 & 0 & 0 \\
\hline 2 & 0.00001 & 0 & 0.00001 & 0.00001 & 0.00001 & 0 & 0 & 0 & 0 \\
\hline 3 & 0.00001 & 0.00001 & 0 & 0.00001 & 0 & 0 & 0 & 0.00001 & 0 \\
\hline 4 & 0.00001 & 0.00001 & 0.00001 & 0 & 0.00001 & 0.00001 & 0 & 0 & 0 \\
\hline 5 & 0 & 0.00001 & 0 & 0.00001 & 0 & 0.00001 & 0.00001 & 0 & 0 \\
\hline 6 & 0 & 0 & 0 & 0.00001 & 0.00001 & 0 & 0.00001 & 0 & 0 \\
\hline 7 & 0 & 0 & 0 & 0 & 0.00001 & 0.00001 & 0 & 0.00001 & 0.00001 \\
\hline 8 & 0 & 0 & 0.00001 & 0 & 0 & 0 & 0.00001 & 0 & 0.00001 \\
\hline 9 & 0 & 0 & 0 & 0 & 0 & 0 & 0.00001 & 0.00001 & 0 \\
\hline
\end{tabular}

\subsubsection{Optimal Path Identification from Pheromone Table}

OP is detected from pheromone concentration which is given in table 2. For choosing the optimal path we just have to look at the edges (table block) with a maximum pheromone concentration starting from sensor 1 (in row 1) till we reach sensor 9. So from $1^{\text {st }}$ node pheromone concentration is maximum in the $3^{\text {rd }}$ column $\left(3^{\text {rd }}\right.$ column in $1^{\text {st }}$ row) which is 0.0001059 . So edge from 1 to 3 is added. Then from the 3 rd sensor (from $3^{\text {rd }}$ row) look for maximum pheromone value. $8^{\text {th }}$ column value is 0.0001138 which is the maximum. So add edge from 3 to 8 . With the node 8 , the maximum pheromone concentration is 0.001153 , so edge from 8 to 9 is added. By proceeding this way one can find a path. In Figure 3 the path with the maximum pheromone concentration is marked with red colour. This path is optimal path. From table 2 it is clear that the pheromone concentration on the path 1 to 3,3 to 8,8 to 9 is higher. So our optimal path is the 1-3-8-9.The length of this path is 85 . We can also get a parallel path if we initially choose $2^{\text {nd }}$ highest node from node $1 \&$ then keep choosing highest pheromone node. The other parallel path proposed by the implemented algorithm OPI using ACO is 1-2-4-5-6-7-9. This path is not optimal but it is sub optimal. This suboptimal path selection is useful when an optimal path for communication is required to be chosen between the communicating nodes. Especially, when one optimal path is over utilised and there is a need to conserve energy of nodes on that path. The length of suboptimal path is 102 . Table 3 gives the number of ants choosing the sensor nodes. Where left column and top row denote nodes. Here $\mathrm{N}$ in top most left block in table 3 stand for node. The number of Ants choosing a path is proportional to the pheromone concentration on the path. Hence many times the path found in Ant table is the same as the result given by the pheromone value. Instead of choosing edge with the highest ant count, if $2^{\text {nd }}$ highest edge is taken at node 1 , then we get another suboptimal path. That path is 1-2-4-5-7-9.This alternate path is very useful as using the same OP will reduce the energy level of the SN in the path. This enables a dynamic detection of alternative optimal path for communication between the communicating sensor nodes.

Table 2 Pheromone value

\begin{tabular}{|l|l|l|l|l|l|l|l|l|l|}
\hline $\mathbf{M}$ & $\mathbf{1}$ & $\mathbf{2}$ & $\mathbf{3}$ & $\mathbf{4}$ & $\mathbf{5}$ & $\mathbf{6}$ & $\mathbf{7}$ & $\mathbf{8}$ & $\mathbf{9}$ \\
\hline $\mathbf{1}$ & 0 & 0.0000353 & $\mathbf{0 . 0 0 0 1 0 5 9}$ & 0.000015 & 0 & 0 & 0 & 0 & 0 \\
\hline $\mathbf{2}$ & 0.0000353 & 0 & 0.0000185 & 0.0000317 & 0.0000166 & 0 & 0 & 0 & 0 \\
\hline $\mathbf{3}$ & 0.0001059 & 0.0000185 & 0 & 0.0000205 & 0 & 0 & 0 & $\mathbf{0 . 0 0 0 1 1 3 8}$ & 0 \\
\hline $\mathbf{4}$ & 0.000015 & 0.0000317 & 0.0000205 & 0 & 0.0000221 & 0.0000158 & 0 & 0 & 0 \\
\hline $\mathbf{5}$ & 0 & 0.0000166 & 0 & 0.0000221 & 0 & 0.0000227 & 0.0000216 & 0 & 0 \\
\hline $\mathbf{6}$ & 0 & 0 & 0 & 0.0000158 & 0.0000227 & 0 & 0.0000208 & 0 & 0 \\
\hline $\mathbf{7}$ & 0 & 0 & 0 & 0 & 0.0000216 & 0.0000208 & 0 & 0.0000145 & 0.000309 \\
\hline $\mathbf{8}$ & 0 & 0 & 0.0001138 & 0 & 0 & 0 & 0.0000145 & 0 & 0.001153 \\
\hline $\mathbf{9}$ & 0 & 0 & 0 & 0 & 0 & 0 & 0.000309 & 0.001153 & 0 \\
\hline
\end{tabular}


Table 3 Ant count

\begin{tabular}{|l|l|l|l|l|l|l|l|l|l|}
\hline & 1 & 2 & 3 & 4 & 5 & 6 & 7 & 8 & 9 \\
\hline 1 & 0 & 42 & 63 & 15 & 0 & 0 & 0 & 0 & 0 \\
\hline 2 & 0 & 0 & 11 & 35 & 20 & 0 & 0 & 0 & 0 \\
\hline 3 & 0 & 12 & 0 & 12 & 0 & 0 & 0 & 69 & 0 \\
\hline 4 & 0 & 14 & 16 & 0 & 21 & 14 & 0 & 0 & 0 \\
\hline 5 & 0 & 1 & 0 & 3 & 0 & 22 & 28 & 0 & 0 \\
\hline 6 & 0 & 0 & 0 & 2 & 12 & 0 & 23 & 0 & 0 \\
\hline 7 & 0 & 0 & 0 & 0 & 3 & 7 & 0 & 13 & 36 \\
\hline 8 & 0 & 0 & 5 & 0 & 0 & 0 & 8 & 0 & 69 \\
\hline 9 & 0 & 0 & 0 & 0 & 0 & 0 & 0 & 0 & 0 \\
\hline
\end{tabular}

\subsection{Comparison of Results with Traditional ACO}

Traditional ACO is already described in this paper. The comparison of results of these two algorithms is given below. The initial pheromone concentration of Traditional ACO is in the table 1. After $5^{\text {th }}$ iteration the pheromone concentration is as shown in table 4 .From table 4 , it is clear that pheromone concentration is more on the path 1-2-4-5-6-7-9.The length of this path is 102. So ants will start preferring this path. In the result section 6 by modified ACO we have found an optimal path which is equal to 85. So it proves that our modified algorithm outperforms the traditional ACO. Figure 4 shows path found by traditional ACO with red colour.

Now the same method is applied to more complex scenario shown in figure 5. In this scenario number of SN are more \& they are densely distributed. Here aim is to find the Multiple Optimal Paths MOP between BS node 1 and SN 30. The modified ACO method which is described previously is used for finding the single Optimal Path. While the optimising number of hops and path length from SN to BS is considered. So these OP are having less path length as well as number of hops. After each iteration, if ants are converged to the single path then that path is selected as the optimal path. As our aim is to find the multiple optimal paths the path which is discovered in previous iteration is deleted from the graph so ants in this iteration will not be able to travel through that path. Deletion of the optimal path in the previous iteration causes ACO to search for the new optimal path in this iteration. In this way several optimal paths are discovered.

Table 4 Pheromone value for Traditional ACO

\begin{tabular}{|l|l|l|l|l|l|l|l|l|l|}
\hline \multicolumn{1}{|l|}{} & 1 & 2 & 3 & 4 & 5 & 6 & 7 & 8 & 9 \\
\hline 1 & 0 & 11.462 & 0.3922 & 0.4440 & 0 & 0 & 0 & 0 & 0 \\
\hline 2 & 11.462 & 0 & 0.6559 & 10.930 & 0.3302 & 0 & 0 & 0 & 0 \\
\hline 3 & 0.3922 & 0.6559 & 0 & 1.066 & 0 & 0 & 0 & 1.564 & 0 \\
\hline 4 & 0.4440 & 10.930 & 1.0663 & 0 & 4.457 & 1.422 & 0 & 0 & 0 \\
\hline 5 & 0 & 0.3302 & 0 & 4.457 & 0 & 13.41 & 1.257 & 0 & 0 \\
\hline 6 & 0 & 0 & 0 & 1.422 & 13.414 & 0 & 3.530 & 0 & 0 \\
\hline 7 & 0 & 0 & 0 & 0 & 1.2572 & 3.53 & 0 & 0.539 & 8.275 \\
\hline 8 & 0 & 0 & 1.564 & 0 & 0 & 0 & 0.539 & 0 & 1.799 \\
\hline 9 & 0 & 0 & 0 & 0 & 0 & 0 & 8.275 & 1.799 & 0 \\
\hline
\end{tabular}




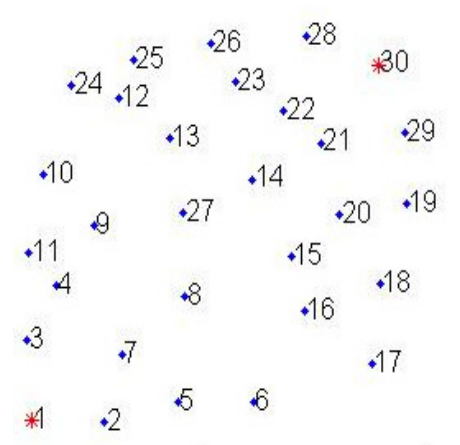

Fig. 5 Wireless sensor nodes and its network

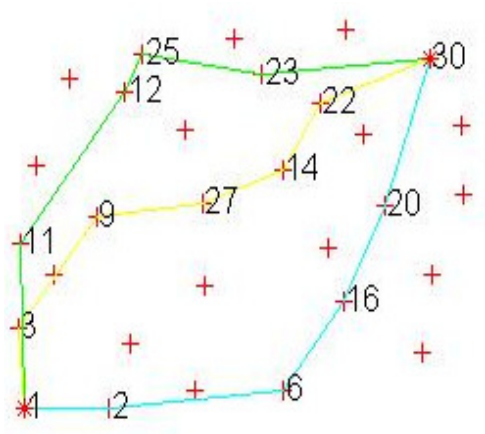

Fig. 6 MOP using modified ACO

Figure 6 shows collection of different optimal and suboptimal paths with different colours. This algorithm is applied for number of SN between 50 to 100. Every time we have got Multiple Optimal paths as described.

\section{CONCLUSION}

Finding the optimal path in dynamically changing resource constrained WSN is challenging. Our work proposes an approach to identify multiple optimal paths for communication between SN to BS. Existing greedy approach is static in providing optimal path. In our computational approach and communication overhead is reduced as BS takes the responsibility of computation \& optimal path is calculated based on pheromone concentration. Multiple optimal paths between SN and BS are useful in forwarding data, efficiently distributing the load. This increases the throughput and increases the network lifetime.

\section{REFERENCES}

[1] Selcuk Okdem and Dervis Karaboga, "Routing in Wireless Sensor Networks Using an Ant Colony Optimization (ACO) Router Chip”, Sensors 2009, 9, 909-921;doi: 10.3390/s90200909

[2] P.K.Singh, Narendra pal singh, "Data Forwarding in Ad-hoc Wireless Sensor Network Using Shortest Path Algorithm", Volume 2, No. 5, May 2011 Journal of Global Research in Computer Science

[3] Adamu Murtala Zungeru, Li-Minn Ang, S.R.S. Prabaharan, and Kah Phooi Seng, "Ant Based Routing Protocol for Visual Sensors”, A. Abd Manaf et al. (Eds.): ICIEIS 2011, Part II, CCIS 252, pp. 250-264, 2011. , Springer-Verlag Berlin Heidelberg 2011.

[4] Selcuk Okdem, Dervis Karaboga "Routing in Wireless Sensor Networks Using Ant Colony Optimization", Proceedings of the First NASA/ESA Conference on Adaptive Hardware and Systems (AHS'06) 0-7695-2614-4/06 2006 IEEE

[5] Dilpreet kaur, Dr.P.S Mundra, "Ant Colony Optimization: A Technique Used For Finding Shortest Path", International Journal of Engineering and Innovative Technology (IJEIT) Volume 1, Issue 5, May 2012

[6] Masaya Yoshikawa, Kazuo Otani, “ Ant Colony Optimization Routing Algorithm with Tabu Search", Masaya Yoshikawa, Kazuo Otani.

[7] Jamal N. Al-karaki, Ahmed E.Kamal, "A Taxonomy of Routing Techniques in Wireless Sensor Network", in Sensor Network Protocols Taylor \& Francis page 3-1 to 4-24 [8] Gizem, Aksahya \& Ayese, Ozcan (2009) Coomunications \& Networks, Network Books, ABC Publishers. 
International Journal of Wireless \& Mobile Networks (IJWMN) Vol. 5, No. 5, October 2013

[8] W. R. Heinzelman, A. Chandrakasan, and H. Balakrishnan, "Energy-Efficient Communication Protocol for Wireless Microsensor Networks", proc. Hawaii International Conference on System Sciences, Vol. 8, pp. 1-10, Jan. 2000.

[9] C.Intanagonwiwat, R.Govindan, and D.Estrin, "Directed diffusion for wireless sensor network", IEEE /ACM transaction . Networking,11(1),2-16,2003

[10] J.Kulik, W.R.Heinzelman, H.Balakrishnan, "Negotion based protocol for disseminating information in wireless sensor network", Wireless Networks, 8, 169-185,2002

[11] M. Dorigo , G. Di Caro, “Ant colony optimization: a new meta-heuristic”, Proceedings of the 1999 Congress on Evolutionary Computation, Proceedings, 1999 